Revue internationale P.M.E.

Économie et gestion de la petite et moyenne entreprise

\title{
Dynamique industrielle et stratégie des PME dans l'industrie des viandes
}

\section{Daniel Barré}

Volume 12, numéro 1-2, 1999

URI : https://id.erudit.org/iderudit/1008654ar

DOI : https://doi.org/10.7202/1008654ar

Aller au sommaire du numéro

Éditeur(s)

Presses de l’Université du Québec

ISSN

0776-5436 (imprimé)

1918-9699 (numérique)

Découvrir la revue

Citer cet article

Barré, D. (1999). Dynamique industrielle et stratégie des PME dans l'industrie des viandes. Revue internationale P.M.E., 12(1-2), 151-170.

https://doi.org/10.7202/1008654ar
Résumé de l'article

Uindustrie de la viande, pour faire face à la crise économique, poursuit sa concentration. Les entreprises développent des stratégies de volume et de différenciation afin de renforcer leur compétitivité dans un marché stagnant et très concurrencé. La logique industrielle et financière des groupes privilégie la croissance externe et l'internationalisation ; les PME, généralement plus ancrées dans un système local de production, recherchent des micromarchés. La filière des produits carnés est dorénavant dominée par son aval et la pression de la grande distribution s'exerce de manière croissante : spécialisation des transformateurs, impératifs de la qualité hygiène-sécurité des produits livrés, innovation permanente. Les PME doivent anticiper sur cette dynamique industrielle dont les contraintes sont de plus en plus pressantes. 


\title{
Dynamique industrielle et stratégie des PME dans l'industrie des viandes
}

\author{
Daniel BARRÉ \\ École nationale d'ingénieurs des travaux agricoles de Clermont-Ferrand
}

\section{MOTS CLÉS}

\section{Stratégie entreprise - PME - Produits carnés - Industrie Gestion - Filière - Distribution des produits alimentaires}

\begin{abstract}
RÉSUMÉ
L'industrie de la viande, pour faire face à la crise économique, poursuit sa concentration. Les entreprises développent des stratégies de volume et de différenciation afin de renforcer leur compétitivité dans un marché stagnant et très concurrencé. La logique industrielle et financière des groupes privilégie la croissance externe et l'internationalisation; les PME, généralement plus ancrées dans un système local de production, recherchent des micromarchés. La filière des produits carnés est dorénavant dominée par son aval et la pression de la grande distribution s'exerce de manière croissante: spécialisation des transformateurs, impératifs de la qualité hygiène-sécurité des produits livrés, innovation permanente. Les PME doivent anticiper sur cette dynamique industrielle dont les contraintes sont de plus en plus pressantes.
\end{abstract}

\begin{abstract}
The meat industry, being confronted to the economic crisis, goes on concentrating. Firms develop volume strategies and try to diversify their production in order to reinforce their efficiency on a rather stagnating and keenly competitive market. In terms of industrial and financial development, groups first insist on external growth
\end{abstract}

\section{L'AUTEUR}

Daniel Barré est maître de conférences à l'École nationale d'ingénieurs des travaux agricoles. II enseigne la gestion et assure la responsabilité de l'option de troisième année: qualité et commercialisation alimentaire. Ses activités de recherche sur la gestion des industries de transformation des viandes s'effectuent dans le cadre du pôle viande clermontois qui associe notamment l'ADIV, l'INRA et le Cemagref, l'Université, le LEGTA et l'ENITA. Adresse: Marmilhat, 63370 Lempdes, France. Téléphone : 3304739813 32. Télécopieur: 330473981390 
and internationalisation. Small and middle-sized firms which are deeply rooted in local production networks are looking for micromarkets. Supermarkets, and more generally downstream pressure is now stronger on the meat products channel: processing industries specialise, finished products and new products present a high safety and hygiene quality. SMEs must anticipate on this industrial dynamic for which the constraints are more and more pressing.

\section{RESUMEN}

La industria de la carne, confrontada a la crisis economica sigue su concentración. Las empresas desarollan unas estrategicas de volumen y de diferenciación para potenciar su competencia en un mercado estancado y competido. La logica industrial y financiera de los grupos hace favor a el crecimiento externo y a la internacionalización. Las PyMEs generalmente mas aferradas en un sistema de producción local buscan unos micro-mercados. La tramitación de los productos a base de carne esta desde ahora dominada por su aval y por la presión que ejercen mucho los supermercados : especialización de los transformadores, imperativos de la calidad higiene-securidad de los productos entregados, inovación permanente. Las PyMEs se deben de anticipar a esta dinamica industrial cuyas obligaciones estan cada vez mas fuertes.

\section{ZUSAMMENFASSUNG}

Die von der Wirtschaftskrise geschüttelte Fleischindustrie setzt ihre Konzentration fort. Die Unternehmungen entwickeln Mengen- und Differenzierungsstrategien, um ihre Wettbewerbsfähigkeit auf einem stagnierenden und hart umkämpften Markt zu verstärken. Die industrielle und finanzielle Situation von Unternehmungsgruppen begünstigt externes Wachstum und Internationalisierung. KMU - in der Regel fest in einem lokalen Produktionssystem verankert - setzen auf Mikromärkte. Der Absatz von Fleischprodukten wird fortan von den Absatzkanälen und dem wachsenden Druck der Grossverteiler bestimmt : Spezialisierung der Verarbeiter, Qualitätsvorschriften im Hygiene- und Sicherheitsbereich für die ausgelieferten Produkte, fortlaufende Innovation. Die KMU müssen dieser industriellen Dynamik vorgreifen, denn der Druck wird zunehmends grösser.

\section{Introduction}

L'internationalisation des marchés, l'apparition de nouvelles formes de concurrence, l'amenuisement des gains de productivité et l'émergence d'une nouvelle trajectoire technologique ont radicalement remis en cause les compromis sociaux de la période des années 1960. La crise économique a engendré, en Europe, des déséquilibres qui s'aggravent. Le chômage augmente; les inégalités sociales et géographiques s'amplifient; les reconversions industrielles se révèlent difficiles. L'expansion de type fordiste est stoppée (Boyer et Durand, 1993). 
Pour le système agroalimentaire communautaire, les effets sont contrastés. L'agriculture est devenue compétitive avec un degré d'auto-approvisionnement atteint pour la plupart des produits agricoles. Le coût de l'alimentation est relativement moins élevé que pour d'autres pays tiers comparables. Mais cette même politique agricole productiviste a engendré un développement inégal à la fois entre les agriculteurs et entre les régions, l'accentuation de l'exode rural, des menaces pour l'environnement, une angoisse chez les consommateurs à l'égard du progrès non maîtrisé. La crise récente de la «vache folle » est de ce point de vue très révélatrice.

Le secteur agroalimentaire subit de fortes contraintes sectorielles. En amont, les fluctuations des matières premières en quantité, prix et qualité s'ajoutent aux nécessités de maîtriser de nouveaux savoir-faire. En aval, la pression de la grande distribution, la prise en compte de la qualité «hygiène-santé » et la stagnation en volume de la consommation alimentaire sont des contraintes partagées par l'ensemble des exploitants. C'est une véritable crise de mutation à laquelle est soumis ce secteur : métamorphose de la consommation, bouleversement des structures des filières et des marchés, évolutions technologiques (Malassis, 1979; Nefussi, 1989). Les industries agroalimentaires (IAA) doivent innover au regard des produits, mais surtout sur la plan des concepts d'alimentation qui anticipent les évolutions socioéconomiques (alimentation, loisirs, santé et culture). À l'égard de la viande, les attentes traditionnelles des consommateurs concernent la tendreté, les aspects diététiques et nutritifs, la facilité d'emploi du produit et un rapport qualité / prix jugé acceptable. Mais l'aspect naturel (sans hormones), la garantie d'origine et les conditions d'élevage deviennent, dans un contexte de crise et de suspicion, des éléments de choix fondamentaux.

Quelle que soit la taille des entreprises, les orientations stratégiques reposent dorénavant sur le triptyque : renforcement de la compétitivité, obtention de résultats, maintien de l'indépendance juridique et financière. Face à cette nouvelle donne économique, quels sont les enjeux et les formes de développement mis en œuvre par les PME du secteur des produits carnés ? Peut-on discerner des stratégies de base chez les groupes et leurs concurrentes de taille plus réduite ? Quelles sont les conséquences pour les nombreuses PME appartenant à cette filière viande en cours d'industrialisation?

Dans la première partie de cette contribution, nous rappellerons (succinctement) les contraintes économiques et spécifiques qui pèsent sur l'ensemble des entreprises. Celles-ci conditionnent, pour partie, les stratégies mises en œuvre par les acteurs. Nous évoquerons ensuite la dynamique industrielle impulsée par l'aval en retenant le cas exemplaire de la filière bovine. La spécialisation et la concentration, associées aux changements du comportement alimentaire des consommateurs, ont naturellement modifié le tissu des entreprises en réduisant sensiblement les PME (et TPE) : 
éleveurs, négociants et bouchers détaillants en particulier. Enfin, nous exposerons les différentes stratégies des entreprises qui s'ordonnent autour du produit, du territoire et de la filière ${ }^{1}$. De toute évidence, l'effet taille est un élément de différenciation, mais là encore, on ne relève aucune loi mécanique d'évolution. La diversité des trajectoires reste la règle.

\section{Des contraintes sectorielles partagées par l'ensemble des entreprises}

La crise économique a particulièrement affecté le secteur des produits carnés avec un certain nombre de caractéristiques et de conséquences qui conditionnent l'environnement des entreprises. Sur le plan macroéconomique, la désorganisation des marchés mondiaux rend les firmes plus vulnérables (Boutonnet et Simier, 1995). La grande dispersion des flux internationaux d'échange, les limites de consommation des viandes atteintes dans les pays riches, l'émergence de nouveaux pays exportateurs et la libéralisation des échanges symbolisée par les derniers accords du GATT sont autant de facteurs déstabilisants pour les opérateurs de la filière.

Sur le plan microéconomique, la restructuration industrielle des entreprises françaises est encore considérée comme insuffisante par les institutions et les organisations interprofessionnelles. Les explications proposées sont nombreuses : taille moyenne des unités de production, surcapacité et éparpillement des outils de transformation, fragilité économique et financière des entreprises de viandes. La faiblesse des politiques commerciales et marketing mises en œuvre amplifie la stagnation-régression de la consommation globale des produits carnés. L'insuffisance du taux de valeur ajoutée de ce secteur ne permet pas de compenser l'importance des frais de personnel. Il en résulte une faible marge nette pour l'industrie de la charcuterie, de l'abattage et de la découpe de bétail. Plusieurs facteurs expliquent la rentabilité étroite de ces industries agroalimentaires à base de main-d'œuvre (Barré, 1997). La production est difficilement automatisable, la matière première est évolutive et périssable. Les cours d'achat des approvisionnements sont fluctuants, la rentabilité est souvent irrégulière et dépendante des aléas conjoncturels.

Par ailleurs, le processus d'industrialisation est caractérisé par des variables dont certaines apparaissent spécifiques à ce secteur d'activité. L'environnement relève d'un contexte général très traditionnel avec un éparpillement d'entreprises à taille réduite ( $70 \%$ de l'effectif total ont entre 10 et 49 salariés). Le caractère «perturbant» des abattoirs publics dans la filière des animaux de boucherie est souvent considéré comme un facteur de distorsion de concurrence. Le surdimen-

1. Programme de recherche 1997-1998, convention FEOGA no 399-97 (objectif 5B Massif central) : Évaluation du potentiel de développement des PME viandes bovines du Massif central. 
sionnement des outils de production est une cause d'abaissement de la rentabilité des investissements. La division du travail croît dans chaque filière, mais elle est inégale selon les sous-secteurs d'activité (porc, volaille, bovins). L'évolution vers des métiers différenciés reste très progressive. Une gamme de produits étendue, régionalisée et traditionnelle est certes une source de richesse collective, mais elle est aussi, selon certains responsables, un frein à la concentration du secteur (difficulté de concilier productivité et économie de variété).

Toutefois, et malgré ses faiblesses structurelles, le secteur industriel des viandes s'est développé au point de se hisser à la première place des IAA françaises avec le chiffre d'affaires le plus important, plus du tiers du nombre d'entreprises et près de 100000 salariés.

\section{Une dynamique industrielle impulsée par l'aval}

L'influence des liaisons entre technologie, produit et marché sont les éléments déterminants de l'industrialisation de la filière des produits carnés (Coquard, 1981 ; Soufflet, 1989). Dans ce secteur, la dynamique industrielle s'est réalisée sous l'impulsion de la grande distribution. Cette dernière a progressivement exigé des industriels la fourniture de biens standard, «marqués » et innovants avec une attente constante de produits réguliers au moindre prix. La valeur d'usage des biens est dorénavant prioritaire sans augmenter pour autant la valeur d'échange. Plus récemment, après que le niveau de saturation de consommation ait été atteint, la sécurité des approvisionnements est devenue un impératif supplémentaire. Avec la montée du consumérisme et l'intégration du secteur dans l'économie de la norme (type ISO), la grande distribution a fait de l'élimination des risques de contamination des aliments un élément prioritaire de référencement de leurs fournisseurs. La qualité et la sécurité se sont transformées en droit de produire. Cette évolution graduelle s'est réalisée en plusieurs phases où ont alterné crise et régulation.

Durant les années 1970-1980, la principale préoccupation des pouvoirs publics est encore de juguler l'inflation. Le régime de taxation de la viande est un élément du contrôle des prix. Les marges bénéficiaires font l'objet d'une étroite surveillance, car la viande représente un tiers des dépenses alimentaires et l'indice des prix sert de référence pour l'indexation des salaires. À l'époque, les crises sectorielles, dont celles de la viande bovine, sont jugées conjoncturelles et la manifestation d'une simple turbulence d'une économie globalement prospère. La croissance résorbe les déséquilibres. On compte alors un grand nombre d'abattoirs publics, assez peu d'abattoirs industriels. Cette structure économique (Bignon, 1974) est caractérisée par l'existence (souvent conflictuelle) d'un grand nombre de «très petites entreprises ». En amont, des groupements de producteurs collectent et trient les animaux issus des éleveurs (985 000 TPE) mais l'organisation de l'offre est encore fragmentaire. Les transactions sur les marchés sont majoritairement 
réalisées par les négociants en bestiaux (7000 TPE) et les commissionnaires (500 TPE). Leur nombre va décroître très sensiblement mais leur place dans la mise en marché des animaux vifs restera toujours importante dans le secteur bovin. En aval, les bouchers détaillants (50000 TPE) s'approvisionnent chez les grossistes (ou encore en circuit rural direct). Ils commercialisent $82 \%$ des tonnages, les supermarchés, $16 \%$ « seulement ». Mais la grande distribution est en pleine expansion ; la saturation du réseau des points de vente n'est pas à l'ordre du jour. La loi Royer de 1973 prévoyant une autorisation pour la création de surfaces supérieures à $1500 \mathrm{~m}^{2}$ ne pourra stopper, pour diverses raisons, cet élan. L'occupation de la périphérie des grands centres urbains par la «distribution moderne» est un facteur essentiel du développement de ces magasins. Proximité, prix et libre-service sont ses atouts concurrentiels.

Durant les années suivantes, trois faits d'importance ont lieu et vont influencer, directement ou indirectement, les rapports de force entre les opérateurs de l'aval de la filière : l'offre en production bovine est devenue excédentaire alors que la consommation de viande bovine régresse (1981); la grande distribution tente de regrouper ses centrales d'achat (1984); la liberté des prix est instaurée (1986). C'est une période charnière et décisive pour le secteur. La grande distribution devient majoritaire. Elle va dorénavant commercialiser plus de $50 \%$ des viandes au consommateur. La dynamique de consommation de viande bovine a changé. Les circuits de distribution ont évolué. La filière bovine a basculé d'un système artisanal et commerçant dans un système semi-industriel.

Toutefois, avec le constat d'une des plus faibles concentrations des IAA et une surcapacité générale d'abattage, le secteur est considéré comme dispersé et enlisé selon la terminologie de Porter (1985). Le négoce est encore dominant, l'offre reste inorganisée et peu maîtrisée. La croissance de parts de marché des uns s'effectue au détriment des autres ; c'est le temps des rachats et de la concentration. Mais certaines PME (la frange de l'oligopole) ayant opté pour une niche (produits, clients ou positionnement géographique) démontrent leur capacité de résistance. Dans le secteur des produits frais alimentaires (telle la viande), les facteurs de sécurité des approvisionnements (contractualisation des apports) et la proximité des marchés de consommation (diminution des frais de logistique et coûts de transport) semblent être les éléments les plus déterminants pour la structuration de ces firmes dans un espace local réduit. L'avantage relatif des PME locales se transforme en atout concurrentiel : diminution du coût d'approche des marchandises, image de fraîcheur des produits, service de proximité, notoriété. C'est probablement l'un des obstacles à la concentration industrielle du secteur viande : pour quelques produits frais, les économies d'échelle sont amoindries par les coûts de stockage et les délais impératifs de livraison des marchandises. La concurrence de certains spécialistes régionaux peut alors s'exercer dans cet interstice de rentabilité potentielle. De plus, la possibilité d'utiliser des abattoirs publics constitue véritablement une rente de 
situation pour certaines PME qui peuvent se consacrer entièrement au négoce sans avoir à rentabiliser un investissement lourd, spécifique et peu rentable.

La crise de la « vache folle » (en 1996) aura renforcé l'instabilité de l'environnement et accru la turbulence économique dans les entreprises du secteur des produits carnés. Ses implications ont déstabilisé les «façons de travailler» des opérateurs : chute brutale des commandes, arrêts de certaines importations, désarroi des éleveurs, nouvelles stratégies lancées et promues par les distributeurs, recentrage sur des filières régionales. C'est une nouvelle régulation économique qui se met en place avec des effets contrastés pour les PME. Face au tassement des taux de croissance des marchés alimentaires, les grandes et moyennes surfaces (GMS) cherchent à conserver leur rentabilité. Elles augmentent leur pression sur les fournisseurs (délai, prix, qualité, services associés), elles intègrent de plus en plus de valeur ajoutée client, elles privilégient leurs marques propres. Mais dans ce contexte de concurrence exacerbée, les enseignes sont aussi dans l'obligation de se différencier. La crise de l'encéphalopathite spongiforme bovine (ESB) a démontré qu' un certain nombre de PME sont apparues comme de nouveaux partenaires privilégiés pour cette démarche. Les raisons en sont multiples : antériorité de la pratique qualité (label notamment), image de tradition, retour vers le local avec l'importance du relationnel pour redonner confiance, référence des distributeurs à la notion d'entreprise citoyenne... Les surenchères en exigences de qualité (traçabilité notamment) augmentent les contraintes et maintiennent le maillon de la transformation des viandes dans une «hétérogénéité industrielle ». Cette situation est naturellement favorable aux PME qui savent tirer parti de leurs facultés d'adaptation et de souplesse commerciale. Mais la démarche qualité représente un coût dont le montant peut être rédhibitoire pour certaines entreprises. La mise en conformité avec les règles dominantes est une exigence qui s'impose à tous. La multiplication des cahiers des charges, les coûts de transaction des contrats, les normes obligatoires (agréments, HACCP, etc.) créent, là aussi, une dépendance organisationnelle dont les effets sur les petites entreprises nous semblent aussi importants qu'une dépendance juridique (Curvalle, cité par Torrès, 1997). Le choix d'une stratégie opportune est rendu d'autant plus nécessaire.

\section{Des stratégies de base différentes selon les entreprises}

«Les options stratégiques de la PME sont avant tout des choix destinés à maximiser les chances de survie de l'entreprise. » (Saporta, 1990) Cette appréciation est particulièrement pertinente pour le secteur des viandes où la vulnérabilité des entreprises s'est fortement accrue. On observe une dualité dans les familles de produits carnés (Despouy, 1993) qui correspond à l'évolution générale des comportements alimentaires : développement des aliments services (praticité, facilité, gain de temps) et croissance des aliments festifs (le plaisir, le gustatif, les racines). Selon les destinations finales, on distingue ainsi : 
- des produits basiques et apporteurs de protéines (steak haché, saucisses, poulet standard...) relativement maîtrisés sur le plan technologique; l'avantage concurrentiel des entreprises de transformation est fourni par une réduction unitaire des coûts de fabrication (stratégie de production de masse) ; la commercialisation s' appuie sur le développement de marques nationales fortes. Ce sont naturellement les groupes qui sont dominants dans ces activités ;

- des «produits nobles» (viandes piécées de boucherie haut de gamme, jambon sec, poulet sous label, etc.) font appel aux qualités organoleptiques (texture, saveur, couleur, etc.). L'avantage concurrentiel est plus difficile à définir : capacité à imposer un différentiel de prix de vente, politique de signes de qualité, développement d'un marketing produit festif, etc. Filiales spécialisées et PME indépendantes se partagent ce secteur des produits de haute qualité gustative. Certains économistes considèrent que les produits nobles et le terroir sont le terrain privilégié pour des PME agroalimentaires spécialisées. Les «très grandes firmes vont devoir cohabiter avec les micromarchés, sources de positionnement et de "niches" pour des PME-PMI fortement ancrées dans leur terroir mais gérées de façon très performantes » (Rastoin, 1995).

Dans le cadre de cette évolution duale de la consommation, les entreprises ont à choisir ou à combiner deux stratégies majeures pour segmenter leurs marchés.

\section{Des stratégies de domination par les coûts}

Dans ce cas, la création et la restructuration des outils de production s'effectuent selon les priorités suivantes : développement d'outils de grande dimension, productifs, modulaires et évolutifs. La mise en place de procédures et de garanties d'hygiène (concept de l'ultrapropre, réalisation des "salles blanches») correspond aux objectifs d'une qualité-sécurité imposée par l'aval des industriels (et par les pouvoirs publics). L'innovation produit est centrée sur la recherche de ruptures technologiques, soit à travers les procédés de fabrication (technologies et biotechnologies) ou des innovations commerciales associant technologie et marketing. La diminution des coûts doit se traduire par une compétitivité-prix qui assure une domination sur le marché. La chaîne de valeur des avantages concurrentiels repose sur l'approvisionnement (maîtrise des matières premières), la production (courbe d'expérience, flexibilité de l'outil, qualité totale), le développement technologique (innovation produit-procédé $)^{2}$.

2. $C f$. diverses études sectorielles réalisées par les services économiques de la Banque de France. 
Dans le secteur des viandes, et à titre d'exemples, ce sont les stratégies développées par les groupes Socopa dans la production de steaks hachés, de Geo dans la fabrication de charcuteries préemballées pour la grande distribution, de Fleury-Michon ou Herta pour la fabrication industrielle de saucisses de Strasbourg.

\section{Des stratégies de différenciation}

La stratégie de compétitivité par la différenciation-produit s'appuie sur des actions de communication et de promotion destinées à valoriser les différences objectives (être reconnu). La perception du produit et sa qualité doivent induire une fidélisation de la clientèle. L'acceptabilité du surprix de la qualité payé par l'acheteur est conditionnée par des critères d'authentification des produits (marque commerciale, image produit, signes de qualité).

Du fait de l'étroitesse de la demande, ce n'est pas la part de marché qui constitue l'axe privilégié de développement des firmes ayant opté pour cette stratégie. Le maintien d'une rente de situation à caractère monopolistique fournit l'avantage concurrentiel déterminant ; il doit au moins compenser les surcoûts liés à la différenciation du produit. L'exemple des viandes bovines fraîches issues de circuits identifiés est très révélateur de cette option d'une stratégie différenciée. Les PME recherchent une clientèle captive; les groupes revalorisent leur image par rapport à leur production de masse. La démarcation s'effectue sur une meilleure identification des produits proposés aux consommateurs (race et origine de l'animal, alimentation et conditions d'élevage, degré de maturation, durée de conservation, etc.) avec l'apposition d'un signe distinctif:

- lié au respect d'un cahier des charges du label («Veau limousin Blason Prestige », «Bœuf Charollais Terroir ») ou par une certification de conformité («Veau les Vittelliers », «Veau Kerméné »);

- lié à l'engagement d'une marque, soit collective («Bœuf de tradition bouchère »), soit commerciale (marque «Bœuf Charal» du groupe Sogéviandes, marque «Bœuf Val Tendre» du groupe Socopa).

Cette stratégie repose sur un avantage d'image avec une chaîne de valeur sur les fonctions : marketing (attente client, communication, notoriété entreprise), commercial (force de vente), technologie (innovation produit). Mais c'est aussi une différenciation spatiale des produits reposant sur les notions de terroir et de typicité. Ce sont généralement des entreprises de taille moyenne qui ont choisi cette orientation dans le secteur agroalimentaire, mais les grandes entreprises associent aussi les deux grands types de stratégies.

La stratégie produit est certainement le facteur le plus discriminant de la rentabilité des entreprises de viandes. Toutefois, les PME peuvent rechercher d'autres stratégies originales pour se positionner sur un ou plusieurs créneaux spécifiques. 


\section{TABLEAU 1}

\section{Domination par les coûts et différenciation dans les IAA}

\begin{tabular}{|c|c|c|c|}
\hline \multicolumn{2}{|c|}{ Outils de production } & \multirow{2}{*}{$\begin{array}{c}\text { Organisation / Innovation } \\
\text { Stratégie de volume }\end{array}$} & \multirow[t]{2}{*}{ Facteurs clés de succès } \\
\hline & & & \\
\hline $\begin{array}{l}\mathbf{G} \\
\mathbf{R} \\
\mathbf{O} \\
\mathbf{U} \\
\mathbf{P} \\
\mathbf{E} \\
\mathbf{S}\end{array}$ & $\begin{array}{l}\text { * Outil de grande dimension } \\
\text { - productif } \\
\text { - modulaire, évolutif } \\
\text { * Ultrapropre et démarche } \\
\text { de qualité globale } \\
\text { * Productique }\end{array}$ & $\begin{array}{l}\text { * Gestion des flux et } \\
\text { gestion de projet } \\
* \text { Logistique intégrée } \\
* \text { Recherche de rupture } \\
\text { technologique (procédé, } \\
\text { packaging) }\end{array}$ & $\begin{array}{l}* \text { Compétitivité prix / baisse } \\
\text { coûts } \\
* \text { Adéquation amont-outil-aval } \\
* \text { Standardisation des matières } \\
\text { premières et valorisation des } \\
\text { produits finis } \\
* \text { Politique de marque et } \\
\text { communication } \\
* \text { Maîtrise de l'information }\end{array}$ \\
\hline \multicolumn{4}{|c|}{ Stratégie de différenciation } \\
\hline $\begin{array}{c}\mathbf{P} \\
\mathbf{M} \\
\mathbf{E}\end{array}$ & * Outil flexible et adaptable & $\begin{array}{l}* \text { Capacité entrepreneuriale } \\
* \text { Flexibilité stratégique } \\
* \text { Innovation produit } \\
* \text { Industrialisation de recette }\end{array}$ & $\begin{array}{l}\text { * Délais, souplesse, service associé } \\
\text { * Proximité géographique } \\
\text { * Image de marque et typicité } \\
\text { (tradition, terroir) } \\
\text { * Qualité organoleptique } \\
\text { des produits }\end{array}$ \\
\hline
\end{tabular}

Les activités de spécialisation offrent de nombreuses occasions de se différencier. En voici quelques exemples.

Le service pour une clientèle hors grande distribution. Cette PME de type familial a choisi de se spécialiser pour un type de clientèle : la restauration hors foyer collective. Elle s' approvisionne en importation de viandes (carcasses) et en animaux vivants sur les marchés à bestiaux locaux qu'elle fait abattre dans le département. Elle vend ses produits finis, après une procédure administrative d'appel d'offres, aux services de restauration des armées. La gestion du personnel est organisée selon les principes de la firme flexible. Un noyau central interne de salariés stables et qualifiés encadrent et dirigent les activités clés de l'entreprise (cinq personnes en gestion et commercial). Un noyau périphérique de travailleurs disponibles est recruté en formule intérimaire (une quinzaine de salariés en découpe et conditionnement : les tâcherons). L'avantage compétitif est naturellement la souplesse en gestion de production renforcé par la garantie des débouchés acquise pour une année (contrat sous réserve de respecter le cahier des charges). En outre, la référence commerciale (agrément de l'armée) constitue une carte d'entrée dans les autres administrations.

Une politique de produit innovant. Cette entreprise implantée dans le centre de la France pratique la découpe, la transformation et le conditionnement de viandes bovines issues d'un troupeau local. Elle compte un effectif moyen de 25 salariés. Son originalité réside dans la production (avec procédé de fabrication breveté) de 
viande feuilletée surgelée et vendue sous marque commerciale. La distribution surgelée spécialisée représente le canal essentiel. L'origine de la viande est garantie par un label, mais l'innovation concerne surtout le procédé qui assure au produit une bonne sapidité, un faible taux de collagène et de matière grasse. À ce jour, il n'existe pas de produit concurrent, mais l'entreprise ne dispose pas d'une force de vente suffisante, ni d'un budget de communication conséquent pour développer son marché. C'est le facteur limitant de cette démarche originale.

D'autres options peuvent être prises : politique de distribution par création de chaîne de boucherie franchisées, sous-traitance pour un distributeur, spécialisation sur un mode d'approvisionnement des transformateurs, etc. La réussite de ces expériences repose toujours sur la flexibilité de l'outil, la réactivité à la demande et la souplesse dans l'organisation. Mais la bonne intégration dans des microfilières de production constitue également le dénominateur commun.

De manière plus générale et pour une industrie de la viande où la matière première représente l'essentiel du coût final des produits, la fiabilité des approvisionnements en animaux et la localisation dans un bassin de production sont des facteurs discriminants de rentabilité (frais de logistique, ajustement offre-demande, équilibrage et valorisation de l'ensemble des produits). C'est une autre approche du comportement des entreprises en termes de localisation et de filière.

\section{Des PME plus ancrées dans un système productif local et à la recherche de micromarchés : l'exemple de la volaille}

Les stratégies régionales de rentes économiques mises en place par des groupes sociaux désireux de préserver leurs intérêts d'une concurrence extérieure représentent un cas particulier bien connu des systèmes de production. Il est naturellement fait allusion aux filières de production organisées autour de protections juridiques des produits de qualité (label, appellation d'origine contrôlée [AOC] en particulier) ou selon une démarche collective privée (certification produit). Il s'agit bien d'un système avec ses acteurs économiques, ses règles internes de fonctionnement, sa permanence temporelle, sa délimitation géographique et sa propre dynamique d'évolution ${ }^{3}$.

Dans ce cas, la territorialisation des opérateurs économiques correspond à une organisation verticale de l'amont à l'aval (filière), mais des relations partenariales de type horizontal s'établissent également. Ces marchés types de produits agroalimentaires de qualité, qu'on est plutôt tenté de qualifier d'agricoles, correspondent

3. Cf. référence traditionnelle à la version du « district industriel » de Marshall, concept de développement régional endogène actualisé par Becattini (1987). 
alors à des microsystèmes de production, relativement fermés, où les décideurs entretiennent des liens conviviaux et s'entendent sur des règles de fonctionnement «à l'amiable ». En définitive, ils pratiquent déjà une forme de "marché concerté » avec une production commune autour d'un concept de qualité, des réseaux de distribution traditionnels bien référencés, une entraide professionnelle (échange de services, défense commune des intérêts) et un ancrage dans le territoire. Toutefois, dans un espace géographique restreint, une certaine rigidité réduit les occasions de relocalisation des entreprises, celles-ci ne disposant pas de la même «plasticité » que les groupes (Gilly, 1988). La marge de liberté des PME reste réduite dans un espace régional. L'opposition est, là aussi, apparente entre le comportement des groupes disposant d'une mobilité financière et géographique des capitaux, d'une logistique intégrée et centralisée et les PME plus dépendantes de leur terroir et des ressources locales. L'exemple suivant nous le démontre.

Le secteur de la volaille présente une certaine originalité : le pouvoir de décision des différents opérateurs est concentré entre quelques groupes ou entreprises d'abattage qui contrôlent la fourniture de poussins, la fabrication des aliments tandis que la majorité des éleveurs est liée par un contrat d'intégration aux abattoirs. Cette industrie bénéficie de facteurs favorables : approvisionnement abondant, diversifié et à des prix compétitifs, des débouchés en constant développement, un haut niveau de technicité avec notamment une sélection française très performante, une production haut de gamme avec les labels, un dynamisme économique sectoriel ayant porté certaines entreprises à un excellent niveau de compétitivité. Avec le renforcement de la crise économique, le marché de la volaille, produits ultrafrais, est devenu très concurrentiel. Les marges des entreprises se resserrent, les perspectives d'évolution des firmes se différencient selon la taille, le degré d'élaboration des produits et la localisation.

Dans cette configuration de marché d'oligopole à firme dominante, l'appui du groupe national à sa filiale régionale est un facteur discriminant de la rentabilité et de la compétitivité. Sur le plan commercial, la filiale bénéficie d'une politique de communication (marque ombrelle) et de la notoriété du groupe «l'image extérieure ». Une politique exigeante de la qualité, instaurée par la société mère, se traduit par une anticipation concernant les mises aux normes des sites de production. C'est aussi la mise en œuvre d'une conception de la qualité totale en conformité avec les exigences de la grande distribution (certifications produit-entreprise, norme ISO 9002). L'accès aux technologies de pointe renforce la différence avec les PME régionales dont les ressources financières sont plus limitées. Contrairement à d'autres produits carnés tels que le bœuf ou le mouton, l'abattage et la découpe primaire de volailles peuvent y être presque intégralement automatisés ; le recours à la productique est un facteur d'économie de substitution. La diminution de la part des frais de personnel dans la valeur ajoutée augmente ainsi la rentabilité brute de l'entreprise. 
L'avantage compétitif des PME locales semble donc limité mais évident avec la notion de service client et l'acceptation de conditions plus draconiennes en matière de livraison. Du fait de la courte durée de vie des produits volailles en rayon, les abattoirs livrent directement leurs produits aux GMS sans passer par les entrepôts régionaux. Pour la même raison, les livraisons sont fréquentes et les délais d'approvisionnement courts. L'atout de certains transformateurs régionaux de taille moyenne est de posséder une souplesse d'organisation qui leur permet de livrer une commande réalisée la veille au soir et sans condition de quantités minimales, ce que refusent les groupes nationaux. Pour les GMS approvisionnées en direct et qui font appel à ces fournisseurs locaux, c'est aussi un moyen de contrebalancer le poids commercial des groupes nationaux, de se prémunir contre les éventuelles ruptures de stocks et de réaliser un taux de marge intermédiaire entre les "premiers prix » et les marques nationales des grands fabricants.

Les PME peuvent-elles se spécialiser dans la production de haute qualité (label) ? Dans le système volailles et à l'inverse des $\mathrm{AOC}$ fromagères ${ }^{4}$, une politique de production et de ventes régionales de poulets labels par une PME indépendante n'apparaît pas comme un pilier stratégique suffisant pour se maintenir et se développer. La vive concurrence des labels nationaux et l'absence d'une consommation traditionnelle par une population locale ne laissent pas la place à des abatteurs régionaux spécialisés uniquement sur les labels. En définitive, la pérennité de ces PME avicoles repose sur une bonne maitrise de leur métier, un positionnement commercial judicieusement choisi, une fidélisation des rapports commerciaux au sein de leur filière et, dans tous les cas, une "gestion dépouillée et sans fioriture » : faibles frais généraux, flexibilité du travail, compression des coûts.

\section{Effet taille, changement d'organisation et stratégie}

L'analyse précédente fait indéniablement apparaitre l'effet structurant de la taille des entreprises. C'est un critère pour élaborer une classification, en sachant bien qu'elle est nécessairement réductrice de la complexité et de la diversité de la réalité économique. Le seul paramètre de la taille est par ailleurs insuffisant pour appréhender la pertinence des stratégies mises en œuvre : la disparition d'entreprise affecte aussi bien les groupes que les PME et les déficits d'activité sont des faiblesses largement partagées quelle que soit la dimension des sociétés ${ }^{5}$. La typologie proposée (volontairement sans référence à un chiffrage de nombre de salariés) n'induit donc aucun déterminisme d'évolution. Elle est avant tout le reflet d'une observation empirique de comportements et d'un constat de trajectoires d'entreprises.

4. Barré et Lagrange, 1990 et 1991.

5. $C f$. études annuelles Banque de France. Observatoire des entreprises. Collection La situation du système productif. Supplément sectoriel. 


\section{Les groupes leaders}

Un premier ensemble de sociétés agroalimentaires de taille importante, au devenir multinational, à la production fortement diversifiée, recherche une position de leadership avec une stratégie volume-coût (Galliano, Pérez et al., 1996). Elles se constituent un portefeuille de marques commerciales (à valeur bilantielle) afin de conforter leur domination sur les marchés. La croissance externe est le vecteur privilégié de leur développement. L'efficience de ces entreprises agroalimentaires (Galliano, 1998) repose notamment sur la possibilité de «fragmenter les aspects décisionnels, fonctionnels et productifs, au sein d'entités juridiquement autonomes » (les filiales) mais «insérées dans une hiérarchie basée sur les liens financiers qui les relient à un centre de décision ultime » (la maison mère). Le secteur de la volaille est particulièrement représentatif de ce modèle organisationnel.

En outre, l'homogénéisation des marchés des économies industrialisées réduit les différences de modèles de consommation et favorise ainsi ces sociétés leaders. Dans le secteur des viandes, le marché est saturé et l'augmentation des parts de marché des groupes s'effectue donc par croissance externe. Les dernières restructurations sont exemplaires à cet égard : reprise d'Arcadie (unité de produits élaborés) puis de Vital par la firme Bigard en viande bovine, absorption de France-Volaille par le groupe Bourgoin. Une autre voie de développement consiste à créer de nouveaux produits sous marque ( «Charal » par Vital-Sogéviande) ou à établir des accords de coopération entre sociétés nationales (Moy-Park, société irlandaise et Bourgoin, groupe avicole français).

\section{Des entreprises de taille moyenne au devenir incertain}

Ces firmes se concentrent sur un nombre restreint d'activités et sur des marchés nationaux. Souvent à dominante familiale et financièrement fragilisées, elles représentent des occasions d'acquisitions pour les firmes dominantes. La pénétration minoritaire des grands groupes dans leur capital constitue la première étape vers leur prise de contrôle ultérieure. Les facteurs différentiels entre ces entreprises de taille moyenne sont la capacité financière disponible, la largeur de gamme de produits offerts, l'appartenance (ou non) à un groupe, le degré de spécialisation des outils de production. Ces entreprises ont longtemps constitué le vivier d'où émergeaient les groupes multinationaux actuels, car leurs trajectoires stratégiques les conduisaient à rejoindre le groupe des «leaders ». Ainsi, au sein des entreprises du secteur de la charcuterie-salaisonnerie, sous l'effet du changement de la demande des consommateurs et de l'ouverture du marché commun, sont apparues conjointement deux tendances. La séparation progressive des activités d'abattage et de découpe et la spécialisation de la gamme produite avec une stratégie commerciale de grandes marques nationales ont modifié les métiers. Selon certains économistes du secteur, ces mouvements furent mis en œuvre par la partie la plus dynamique et la plus 
novatrice des chefs d'entreprises de PME qui trouvèrent dans cette double spécialisation les conditions d'une croissance plus rapide et plus rentable ${ }^{6}$. Les évolutions des sociétés Prédault et Madrange dans le secteur des jambons cuits ont valeur d'exemple pour ces trajectoires leader-produit.

\section{TABLEAU 2}

Exemple de trajectoires dans le secteur de la charcuterie-salaisonnerie

\section{PHASE 1 - PROFIL TRADITIONNEL}

PME à capital familial

Dirigeant : un patron

Part importante de la viande fraîche commercialisée

Importance des ventes en circuit traditionnel

Polyvalence de l'outil

Qualité : savoir-faire traditionnel

Gestion empirique

Nombreuses références des produits commercialisés

Service de proximité

POINT DE RUPTURE STRATÉGIQUE : ABANDON DE L'AMONT (abattage et découpe de viande)

Remise en cause du système d'approvisionnement

Compensation de la réduction de l'activité de découpe par spécialisation, focalisation...

Évolution technologique (contrôle qualité)

Nouvelle pratique de la gestion

Changement de la politique commerciale (force de vente, communication...)

Acquisition de nouveaux savoir-faire professionnels

\section{PHASE 2 - PROFIL PRÉINDUSTRIEL}

Ouverture du capital de la société

Recentrage de l'activité par métier et marché

Niveau technique croissant, recrutement de cadres extérieurs à l'entreprise

Dimensionnement des ventes

Agrément de l'outil et procédures qualité totale

Maîtrise des flux, industrialisation des recettes

Degré d'informatisation élevé

Augmentation des immobilisations incorporelles (immatériel)

Distribution GMS et restauration hors foyer

Les «moyennes entreprises ont parfois les inconvénients des petites structures, mais pas encore les avantages de la grande dimension ». Dans le secteur des produits carnés, ces entreprises «dilemme » ne pourront se maintenir que si elles se trouvent en aval d'une production quantitative et qualitativement adaptée, avec un regroupement en pôles d'excellence locale (leader régional) ou concentrées sur

6. $C f$. travaux sectoriels et études du cabinet AND (Agriculture Nutrition Développement), Paris. 
un marché spécifique (innovation produit). Dans tous les cas, elles devront développer une gestion très compétitive.

\section{Des PME fragilisées, mais offrant une capacité de résistance à la crise}

Un dernier tissu industriel composé de petites et moyennes sociétés souvent artisanales, de taille réduite, sans marque bien implantée, mais s'appuyant sur un savoir-faire traditionnel, entame des stratégies de concentration sur un segment étroit: la «niche » produit, le positionnement régional très localisé ou la soustraitance, soit pour des fabricants nationaux, soit pour la grande distribution. L'objectif vital est d'assurer la pérennité dans un contexte de concurrence accrue ; à défaut de quoi, c'est la menace de disparition. Le dynamisme de certaines petites entreprises de produits carnés repose sur une opposition apparente au modèle d'évolution dominant :

- recherche d'une taille optimale, mais pas nécessairement maximale (adaptation de la taille critique aux perspectives réelles du marché);

- souplesse dans les approvisionnements et sans les contraintes de volume ;

- refus d'une spécialisation à outrance ;

- rejet de l'externalisation de certaines fonctions (la détention d'un parc de transport est clairement ressentie comme un avantage concurrentiel: disponibilité, service, relationnel, etc.);

- modernisation et investissement modérés, utilisation de matériels amortis ;

- déclinaison d'une gamme de produits autour d'une même technologie;

- pas (ou peu) de force de vente et de communication;

- contrôle maîtrisé des frais et du taux d'endettement;

- culture de l'indépendance commerciale et financière.

Comme pour d'autres secteurs industriels (Paranque, 1994), ces entreprises sont moins capitalistiques que leurs concurrentes de grande taille; elles gèrent «en bon père de famille ». Dans une conjoncture difficile (du fait des crises cycliques dans la production animale ou de la réduction de la consommation), elles font preuve d'une grande capacité de résistance alors que leurs homologues intermédiaires «modernisées, spécialisées et endettées » montrent une fragilité financière et commerciale plus sensible aux aléas des marchés. Il paraît délicat, au stade actuel d'industrialisation de la filière viande, d'apprécier la portée réelle de ce comportement atypique. Assistons-nous à l'émergence d'entreprises d'avant-garde ou à des formes résiduelles et artisanales d'adaptation à la mutation économique ? Plus généralement, la crise est-elle un élément de regain des PME ? (Ganne, 1994) 


\section{Conclusion}

Les PME constituent un pôle important de l'agroalimentaire, elles disposent d'avantages concurrentiels indéniables, mais fragiles. Dans le secteur de l'industrie des viandes, les PME régionales occupent en réalité des créneaux commerciaux risqués et instables : produits marginaux à faible valeur ajoutée, à taux de service élevé, coût des tournées de distribution, importance des circuits de vente en régression (grossistes, détaillants et boucheries traditionnelles), métiers très spécifiques. Cependant, nous l'avons évoqué, c'est justement de la bonne gestion de ces contraintes et des opportunités du marché que résulte leur avantage concurrentiel. Comme pour d'autres industries (Marchesnay, 1991), notre contribution valide l'hypothèse selon laquelle les microfirmes sont performantes dans des activités à déséconomies d'échelle (activité à forte charge en services) ou à déséconomies de champ (micromarchés fortement segmentés).

Dans le foisonnement de petites et moyennes entreprises du secteur des produits carnés, il y a manifestement des comportements économiques d'acteurs et des mécanismes particuliers de fonctionnement. Ils sont révélateurs d'une utilisation différentielle du capital et des compétences humaines. On peut y entrevoir des convergences avec la remise en cause globale de l'instrumentation de gestion des entreprises et l'émergence de nouveaux principes de fonctionnement : transversalité, polyvalence, gestion de projet, valeur-client, etc. (Affriat, Fleury et al., 1997). Par ailleurs, les théories développées sur la bipolarisation de l'appareil productif, facteur d'inégalité en matière de développement régional, et le regain d'intérêt pour les réseaux de PME, non plus considérés comme une phase de transition vers la grande entreprise, mais comme un phénomène permanent du secteur industriel, valorisent les microsystèmes productifs. Certains économistes y distinguent même un mouvement spécifique d'industrialisation en milieu rural (Quevit et Vandoren, 1993). Pour autant, ce phénomène de persistance d'un tissu de PME remet-il en cause la tendance profonde de l'économie marchande à la concentration et à la constitution de groupes monopolistiques ou n'est-il qu'un épiphénomène d'adaptation transitoire et conjoncturelle à la crise économique ? Une approche sectorielle telle que la nôtre, dont le champ d'investigation est nécessairement restrictif, ne permet pas d'y répondre.

De toute évidence, la prise en compte de la mesure du temps (l'horizon économique et la perspective historique) est un préalable fondamental à toute interprétation des tendances d'évolution. Dans son ensemble, le secteur de la viande est caractérisé par une forme d'organisation préindustrielle où la logique de rationalité taylordienne-fordienne est encore prégnante. Les configurations de marchés sont de type oligopolistique avec une frange concurrentielle composée de multiples PME ; l'intégration verticale y prédomine. C'est une activité agroalimentaire à base de main-d'œuvre et à dominante de coût en matières premières. Industrie à coefficient 
de capital élevé, les gains de productivité portent encore sur la production matérielle et sur l'agencement des facteurs de production. Mais à l'exception des sous-secteurs caractérisés par des rendement croissants, la taille de l'entreprise n'est pas un facteur de compétitivité-prix. L'efficience et la rentabilité sont présentes à la fois dans les plus petites et les plus grandes entreprises. Ce constat n'est pas incompatible avec le processus de concentration en cours de réalisation. En dernière instance, c'est un secteur des produits carnés en voie de mutation et dont la maturité industrielle paraît, selon nous, inachevée. Dès lors, la pertinence et la pérennité des modes d'organisation spécifiques développées par les PME seront jugées sur la capacité à reproduire durablement leur système socioéconomique, et ce, dans un contexte de crises sectorielles successives.

\section{Bibliographie}

AFFRIAT, C., B. FLEURY et al., (1997), Entreprise et performance globale. Outils, évaluation, pilotage, Commissariat Général du Plan, Economica, novembre, p. 256.

BARRÉ, D. (1997), «Contraintes économiques, indicateurs de performance et compétitivité : l'exemple des produits carnés », Revue Industries Alimentaires et Agricoles, décembre, p. 854-858.

Barré, D., J. Giroux, R. Lacabérats, P. Mainsant, C. Potherat et F. Porin (1997), «Consommation des viandes Viandes et Produits Carnés », Revue ADIV, numéro spécial, vol. 18, Clermont-Ferrand, p. 88.

BARRÉ D. et L. LAGRANGE (1990), «La filière des fromages d'appellation contrôlée en Auvergne », Revue d'Auvergne, n 4, p. 115-137.

BARRÉ D. et L. LAGRANGE (1991) «L'impact économique des produits agro-alimentaires de qualité. Le cas de l'Auvergne », Contribution au Colloque CERAMAC, ClermontFerrand, Université Blaise-Pascal, 16-17 septembre 1991, 14 p.

BeCATtini, G. (dir.) (1987), Mercato e forze locali : il distretto industriale, Bologna, II Mulino.

BIGNON, C. (1974), Rapport de la commission d'enquête parlementaire sur la commercialisation des viandes, Rapport $\mathrm{n}^{\circ}$ 1553, Paris, Assemblée nationale, p. 146.

BoutonNET, J.P. et J.P. SimiER (1995), Les viandes, Paris, Economica, Collection « Cyclope poche », $110 \mathrm{p}$.

BOYER, B et J.P. DURAND (1993), L'après fordisme, Paris, Syros.

COQuART, D. (1981), «Processus d'industrialisation de la filière viande bovine », Thèse, Université de Rennes.

DESPOUY, J. (1993), «Viandes bovines, les limites de l'industrialisation », Revue Analyse Financière, octobre, p. 15-21.

EUROSTAF (1994), L'industrie de la viande : modernisation, restructuration, segmentation et concentration, Collection «Analyse de secteur », $280 \mathrm{p}$. 
GANNE, B. (1994), « Les PME dans le système français : heurts et malheurs et mode de gouvernance », dans PME et développement économique en Europe, dirigé par A. Bagnasco et C.F. Sabel, Paris, Édition La Découverte, Collection « Recherche », p. 137-158.

GAlliano, D., R. PEREZ et al. (1996), «Les multinationales de l'agroalimentaires », Économie Rurale, $\mathrm{n}^{0}$ 231, janvier-février, p. 3-70.

GALliano, D., (1998) «Complexité et forme d'efficience des organisation industrielles : le cas des groupes de l'agroalimentaire », Économie appliquée, tome LI, no 1, p. 77-108.

GILLY, J.P. (1988), «L'analyse des systèmes productifs régionaux », Traité d'Économie Industrielle, Paris, Economica, p. 351-369.

HY, M., B. LASSAUT et F. NiCOLAS (1989), «Concurrence entre les formes industrielles et artisanales de production-distribution des biens alimentaires : exemples de la boulangerie et de la boucherie », Revue Économies et Sociétés, Les stratégies agroindustrielles, p. 197-216.

Julien, P.A. et M. MARChesnay (1988), La petite entreprise, Paris, Vuibert Gestion, 288 p.

LAGRANGE, L. (1995), La commercialisation des produits agricoles et alimentaires, Paris, Tec and Doc Lavoisier, $448 \mathrm{p}$.

LOPEZ, E. et J. MUCHNIK (1997), Petites entreprises et grands enjeux. Le développement agroalimentaire local, tome 1, Paris, L'Harmattan, $361 \mathrm{p}$.

MAINSANT, P., G. DE FONTGUYON et al. (1994), La demande du consommateur et l'offre de viande bovine du linéaire boucherie à l'horizon 2000, CODIVIAL-INRA.

MALASSIS, L. (1979), Économie agroalimentaire, tome 1, Économie de la consommation et de la production agroalimentaire, Paris, Cujas, $437 \mathrm{p}$.

MALVY, M. (1991), Rapport de la commission d'enquête relative au fonctionnement du marché de la viande ovine et bovine, Rapport $\mathrm{n}^{0} 1950$, Paris, Assemblée nationale, tome 1, p. 308 ; tome 2, p. 430.

MARChESNAY, M. (1991), «PME : une gestion spécifique?», Économie Rurale, n 206 , novembre-décembre, p. 11-17.

Marshall, A. (1920), Principles of Economics, $8^{\mathrm{e}}$ édition, New York, MacMillan, 731 pages.

NEFUSSI, J. (1989), Les industries agroalimentaires, Paris, Presses universitaires de France, décembre, Paris, 127 p.

PARANQUE, B. (1994), «Fonds propres, rentabilité et efficacité chez les PMI», Revue d'Économie Industrielle, numéro spécial PME-PMI et Économie industrielle, $\mathrm{n}^{\circ}$ 67, $1^{\text {er }}$ trimestre, p.175-190.

PORTER, M. (1985), Competitive Advantage, Traduction française (1986), L'avantage concurrentiel, Paris, InterÉditions, 647 p.

QUEVIT, M. et P. VANDOREN (1993), « Stratégies d'innovation et référents territoriaux », Revue d'Économie Industrielle, $\mathrm{n}^{\circ} 64,2^{\mathrm{e}}$ trimestre, p. 39-53.

RASTOIN, J.L. (1995), « Dynamique du système alimentaire français », Revue Économie et Gestion Agroalimentaire, $\mathrm{n}^{\circ} 36$, juillet, p. 5-14. 
SANS, P. (1996), « Relations verticales et pouvoir au sein du canal de distribution : le cas de l'industrie de transformation de la viande bovine française dans ses rapports avec la distribution moderne », Thèse, Université de Toulouse I, juillet, $270 \mathrm{p}$.

SAPORTA, B. (1990), «Stratégie des petites et moyennes entreprises », Encyclopédie de Gestion, Paris, Economica, p. 2730-2754.

SAVOYE, B. (1994), « La taille des entreprises, élément structurant du système productif », Revue d'Économie Industrielle, $\mathrm{n}^{\circ}$ 67, $1^{\text {er }}$ trimestre 1994, numéro spécial PME-PMI et Économie Industrielle, p. 103-119.

SOUFFLET, J.F. (1988), La filière bétail et viande bovine - Fonctionnement et évolution, Livre 1 : «La filière et l'analyse de la filière » $(165$ p. $)$ Livre $2:$ «La filière bétail et viande bovine, fonctionnement et évolution », Thèse, Université de Montpellier, 670 p.

SOUFFLET, J.F. (1989), « Les stratégies industrielles dans la filière viande bovine » et «Les stratégies agroindustrielles », Économie et Société, Progrès et Agriculture, $\mathrm{n}^{\circ} 20$, juillet, p. 179-196.

TORRÈs, O. (1997) «Pour une approche contingente de la spécificité de la PME, Revue internationale PME, vol. 10, $\mathrm{n}^{\circ}$ 2, p. 9-43. 